\title{
Attitudes towards Mathematics: Effects of Individual, Motivational, and Social Support Factors
}

\author{
Maria de Lourdes Mata, Vera Monteiro, and Francisco Peixoto \\ ISPA, Instituto Universitário, UIPCDE, Rua Jardim do Tabaco 34, $1149-041$ Lisboa, Portugal \\ Correspondence should be addressed to Maria de Lourdes Mata, lmata@ispa.pt \\ Received 11 May 2012; Revised 4 August 2012; Accepted 19 August 2012 \\ Academic Editor: Helga Krinzinger
}

Copyright (C) 2012 Maria de Lourdes Mata et al. This is an open access article distributed under the Creative Commons Attribution License, which permits unrestricted use, distribution, and reproduction in any medium, provided the original work is properly cited.

\begin{abstract}
This paper aims to understand how certain different but interrelated variables such as background, motivation, and social support could lead to an explanation of student attitudes towards math and to an understanding of the defining characteristics of these attitudes in the school environment. Participants consisted of 1719 Portuguese students, from fifth-to-twelfth grade. The study utilizes an adaptation of the "Intrinsic Motivation Inventory" assessing main determinants of intrinsic motivation. One section of the questionnaire- "In my Math Class"-also assesses student perceptions of teacher and peer support as well as student attitudes. The results revealed that, in general, students held positive attitudes towards mathematics and also highlighted the main effects of grade and math achievement on these attitudes. No gender effect was identified although the girls showed a continuous decline in attitudes the further they progressed in school. A hierarchical analysis using structural equation modeling showed that motivationrelated variables are the main predictors of attitudes towards mathematics and that teachers and the social support of peers are also highly significant in understanding these attitudes.
\end{abstract}

\section{Introduction}

Proficiency in languages, science, and mathematics is seen as an essential precursor to success in modern society. In Portugal, recent guidelines, set by the Ministry of Education regarding Mathematics and Portuguese Language curricula, tasks, evaluation, and workload, reflect this concern as these subjects are cross-curricular and are used in daily life. Comparative international evaluations [1] revealed that Portuguese students did not perform as well as expected, and that they underachieved in mathematics and languages when compared to students from other countries in the OECD. In mathematics, results showed that whilst there was an improvement in mathematics performance by Portuguese students from 2003 to 2009, in 2009, on a scale of six levels, Portugal still has in the region of $25 \%$ of their students at level 2 or below [1].

These results give impetus to the development of further research that seeks to characterize and understand different variables which may influence student performance. This will help to make possible strategies for future action in schools, families, and communities, in order to bring about an improvement in the failure rate in math.

The complexity of factors that can influence math performance is demonstrated by Singh, Granville, and Dika [2] when they show that high achievement in mathematics is a function of many interrelated variables related to students, families, and schools. Among student variables, attitudes are regarded by several researchers, as an important/key factor to be taken into account when attempting to understand and explain variability in student performance in maths [3-6].

Mobilizing a set of different definitions concerning attitudes presented since 1935, Eshun [7, page 2] defines an attitude towards mathematics as "a disposition towards an aspect of mathematics that has been acquired by an individual through his or her beliefs and experiences but which could be changed." When emphasizing the importance of individual experiences, the contexts where students interact with others and with mathematics become important focal points. Fraser and Kahle [8] have also highlighted this aspect in research which shows that learning environments at home, at school, and within the peer group accounted for 
a significant amount of variance in student attitudes and, furthermore, that class ethos had a significant impact on the scores achieved by students for these attitudes.

In addition, Mohamed and Waheed [5] when reviewing literature aimed at understanding attitudes and the influences on their development in relation to differences between students, identified three groups of factors that play a vital role in influencing student attitudes: factors associated with the students themselves (e.g., mathematical achievement, anxiety, self-efficacy and self-concept, motivation, and experiences at school); factors associated with the school, teacher, and teaching (e.g., teaching materials, classroom management, teacher knowledge, attitudes towards maths, guidance, beliefs); finally factors from the home environment and society (e.g., educational background, parental expectations).

Attitudes can be seen as more or less positive. A positive attitude towards mathematics reflects a positive emotional disposition in relation to the subject and, in a similar way, a negative attitude towards mathematics relates to a negative emotional disposition [9]. These emotional dispositions have an impact on an individual's behavior, as one is likely to achieve better in a subject that one enjoys, has confidence in or finds useful [7]. For this reason positive attitudes towards mathematics are desirable since they may influence one's willingness to learn and also the benefits one can derive from mathematics instruction [7].

1.1. Attitudes and School Grades. Nicolaidou and Philippou [6] showed that negative attitudes are the result of frequent and repeated failures or problems when dealing with mathematical tasks and these negative attitudes may become relatively permanent. According to these authors when children first go to school they usually have positive attitudes towards mathematics. However, as they progress their attitudes become less positive and frequently become negative at high school. Köğce et al. [3] found significant differences between younger and older students' attitudes towards mathematics with 8th graders having lower attitudes than 6th graders.

There are a number of factors which can explain why attitudes towards mathematics become more negative with the school grade, such as the pressure to perform well, over demanding tasks, uninteresting lessons and less than positive attitudes on the part of teachers [6].

1.2. Gender and Attitudes towards Maths. Gender differences are a recurrent theme throughout the literature in academic studies in general and in math studies in particular. Math is often considered to be a domain in which boys are higher achievers, both in terms of attitudes and self-concept. Contrary to this, findings show that math school achievement and grades do not differ significantly between boys and girls (e.g., $[10,11])$. This similarity in performance between males and females is clear in the meta-analysis conducted by Lindberg et al. [11] with data from 242 studies representing 1.286.350 people, indicating no gender differences $(d=0.05)$ and nearly equal male and female variances.
There are, however, noticeable differences in the beliefs held by boys and girls. Research has consistently shown that girls have lower math self-concept than boys (e.g., [12]). Results concerning gender differences in attitudes are less consistent than those in self-concept. Some studies have reported significant differences when we compare girls and boys attitudes towards mathematics [7, 13-15], nevertheless there are a number of studies where these differences are not identified $[3,5,6,16,17]$. A meta-analysis conducted by Etsey and Snetzler [17] taking into consideration 96 studies $(n=30490)$ concluded that gender differences in student attitudes toward mathematics do exist but are small. The results indicate that males show more positive attitude. However in elementary school studies the effect size was about .20 in favor of females and for grades 9 to 12 the effect size was similar, .23, but in favor of males. Also Hyde et al. [18] in their meta-analysis confirm small gender effects, which increase among older students (high school and college), with females holding more negative attitudes. Although these meta-analyses were developed in the 1990s, there is recent research which confirms these results $[13,14]$ and attempts to provide a justification for it. Asante [13] states that, when compared with boys, "girls lacked confidence, had debilitating causal attribution patterns, perceived mathematics as a male domain, and were anxious about mathematics" [13, page 2]. The research carried out by this author in Ghana, showed that boys had more positive attitudes towards mathematics than girls. Also Sanchez et al. [14] in a study with North American students found significant gender differences in eighth grade students' attitudes towards math. American boys showed more interest in math than girls, but girls perceived math as more important than boys. Girls also presented higher scores on items with regard to difficulties with math. According to Asante [13] school environment, developmental changes in gender identity, and teacher and parent attitudes and beliefs towards mathematics are factors that may contribute to the differences identified between boys and girls in their attitudes towards mathematics.

Nonetheless there is research which concludes that gender does not affect attitudes towards mathematics [3, $5,6,15,16]$. The meta-analysis conducted by $\mathrm{Ma}$ and Kishor [15] which looks at 113 studies $(n=55265)$, when studying the effects of gender, concludes that this variable did not have a significant effect on the relationships between attitudes and performance in mathematics because separate analysis by gender demonstrated similar significant effect sizes. Georgiou et al. [16] showed that there was no difference either in math achievement or in math attitudes between boys and girls. However, high achieving boys and girls, despite both considering math as an attractive subject, differed in the explanations they gave for their performance. Since the ability attributions of boys were higher, they believed that their grades were due to their intelligence more consistently than girls did.

1.3. Achievement in Mathematics and Attitudes. Several studies have been undertaken to try to reach an understanding of the relationship between student attitudes towards 
mathematics and academic achievement $[4-6,8,15,19]$. In $\mathrm{Ma}$ and Kishor meta-analysis [15] only weak correlations between these variables were identified and these relationships were dependent on several variables (e.g., grade, sample size, ethnic background). With regard to grade, these associations become stronger among older students (7th to 12 th grade).

However, more recent studies point to a positive correlation between student attitudes towards mathematics and student academic achievement. Along these lines are the results obtained by Nicolaidou and Philippou [6] which reveal significant correlations between attitudes and performance. Students having positive attitudes achieved better. Mato and De La Torre [4] in a study with secondary school students also showed that those with better academic performance have more positive attitudes regarding math than those with poorer academic performance. These results were confirmed in wider research, concerning math study attitudes among the secondary school students of nine countries, developed by Sanchez et al. [14].

Lipnevich et al. [20] in a study developed with USA and BieloRussian middle school students highlighted the importance of attitudes in predicting academic achievement, when it showed that mathematics attitudes explained a variance of $25 \%$ to $32 \%$ in mathematics achievement, with much of the explained variance independent of ability in math.

Nevertheless, Georgiou et al. [16] showed that high achievement could serve to predict a positive attitude towards math, but such an attitude could not predict stronger achievement. However, these authors emphasize the role of teachers and schools in changing attitudes stating that, math achievement could be improved by, for example, better teaching methods, more motivated teachers or better course books, which has as its corollary the improvement of attitudes towards math.

1.4. Mathematics Learning Environments and Attitudes. Akey's [21] work showed that several aspects of school context (e.g., teacher support, student-to-student interaction, and the academic and behavior expectations of the teacher) were significantly related to student attitudes and behaviors. Akey [21] concluded that the class environment where teachers who students see as supportive promote student feelings of control and confidence in their ability to succeed. The way students perceive teacher characteristics will affect their attitudes towards mathematics [22]. Maat and Zakaria and Vaughan $[22,23]$ identified a significant relationship between learning environment and attitude towards mathematics. Students with a higher perception of the learning environment and a more positive perception of their teachers have more positive attitudes towards mathematics [22]. Rawnsley and Fisher [24] also found that students had more positive attitudes toward mathematics when their teacher was perceived to be highly supportive.

1.5. Motivation and Attitudes. A number of authors have shown that the relationship between aspects of the social environment and student emotional aspects may be mediated by other variables such as control-related appraisals and values-related appraisals $[25,26]$. Therefore, competence support, autonomy support, expectations, and feedback that students receive from others have an impact on their cognitive appraisals and these are the main sources of their emotional dispositions. When studying attitudes, it is important to take into consideration the role of these mediated variables where we can include the motivation features of each student. In this sense, Wigfield [27], in reading specific domain, maintains that attitudes, realized as the individual's feelings towards reading, could be related to the motivation of the individual concerned because they influence how much individuals involve themselves in reading activities. Attitudes are affective responses that accompany a behavior initiated by a motivational state [28]. Attitudes can therefore be linked directly to motivation and provide key information to a better understanding of attitudinal and motivational processes. In the domain of maths there is little research that studies the relationships between motivation and attitudes. However, a number of studies have highlighted some specific associations. Singh et al. [2] used two sets of items to tap motivation, one related to attendance of school and classes and another to participation and preparedness for math classes. The authors concluded that mathematics attitude was affected by motivational factors since significant direct effects of .19 and .21, of these two motivation components were identified in student attitudes. Students who displayed school behavior associated with low motivation (e.g., coming late to school, skipping classes, coming unprepared without books and homework) had a more negative attitude toward mathematics. Other authors have taken into consideration Effort as an indicator of motivation [29, 30]. Reynolds and Walberg [30] using structural equation modeling to analyze diverse factors effects on math's performance and attitudes with 11th grade students, identify a significant effect on motivation in math attitudes. Hemmings and Kay [29] in a study with 10th grade students also verified that Effort was positively and significantly related to math attitudes.

1.6. Objectives. This study has two main objectives: firstly, to analyze the effects on math attitudes of factors usually analyzed in the literature (gender, grade, and achievement) among Portuguese school students; secondly, to analyze the effect on attitudes in this group of other factors that have been less well researched, associated with learning environment (e.g., perceived math's teacher and peers' support) and with the motivational characteristics of students.

\section{Method}

2.1. Participants. 1719 fifth-to-twelfth grade Portuguese students from different schools in Lisbon and the surrounding area participated in the study. They were from a wide range of social and economic backgrounds. There were 869 boys and 850 girls. In Portugal, school grades are organized in four cycles: 1st Cycle (1st to 4th grade), 2nd Cycle (5th and 6th grades), 3rd Cycle (7th to 9th grade), and Secondary (10th 
TABLE 1: Distribution of participants by study cycle.

\begin{tabular}{lcc}
\hline Level & $n$ & $\%$ \\
\hline 2nd Cycle (5th and 6th grades) & 451 & 26.2 \\
3rd Cycle (7th, 8th, and 9th grades) & 847 & 49.3 \\
Secondary (10th, 11th, and 12th grades) & 421 & 24.5 \\
\hline
\end{tabular}

to 12 th grade). In this research participants were from the 2nd and 3rd Cycles and Secondary. The student distribution according to study cycle is presented in Table 1 .

In terms of achievement at mathematics, Portuguese students from the 2th and 3rd Cycles have been evaluated on a five point scale, where 1 and 2 are negative marks, 3 is medium, and 4 and 5 are good or very good marks. Secondary students are assessed on a scale ranging from 0 to 20 that we converted into a 5-point scale similar to those of other cycles. Based on a mean of the two last mathematics evaluations, students in this research were organized in three different achievement groups: Low-with marks lower than $3(27.4 \%)$, Medium - with marks of 3 or $3,5(30.1 \%)$, and Good-whose marks ranging from 4 to $5(42.5 \%)$

2.2. Procedure. Data used in this study was collected at school. Letters describing the study were sent to parents who gave their written consent to the head teacher.

Questionnaires were administered in the classroom under the supervision of a member of our research team. Questionnaires were read aloud for younger students whenever it was thought necessary.

2.3. Instruments. The motivation towards math was measured through a version of IMI Intrinsic Motivation Inventory, directed towards Mathematics, taking into consideration three dimensions: Perceived Competence, Perceived Choice, and Value/Utility [31]. This instrument is conceptualized to take into consideration the main constructs of the Self-Determination Theory (SDT) $[32,33]$. Therefore, the Perceived Choice and Perceived Competence are theorized as positive predictors of intrinsic motivation and are related to the SDT innate psychological needs of autonomy and competence [34]. The Value/Usefulness subscale embodies the idea that people internalize and develop more self-regulatory activities when experience is considered as valuable and useful for them [34].

The questionnaire comprised 14 items, distributed over three dimensions: Perceived Competence-four items (e.g., "I think I am pretty good at Math's activities"); Value/Utility—five items (e.g., "Math's activities are valuable to me"); Perceived Choice-five items (e.g., "I only do the Math's tasks because the teacher orders me to"). All items were scored on a 6-point scale ranging from 1 ("Never") to 6 ("Always"). Items from the Perceived Choice dimension were reversed due to their negative formulation. In this instrument higher scores are related to intrinsic motivation characteristics.

In order to verify the adequacy of item inclusion in the correspondent dimension, reliability has been analyzed using
Cronbach's Alpha. Reliability scores for our sample in the three subscales can be considered adequate as Cronbach's Alpha values were .80 for Perceived Competence, .84 for Perceived Choice, and .93 for Value/Utility [35].

Student perceptions of classroom support (teacher and students support) and attitudes towards mathematics were measured by 16 items extracted from the classroom climate scale "In my Math class" [36]. Teacher Social Support (TSS) is a six item dimension looking at the extent to which students feel that their math teacher supports them (e.g., "My math teacher cares about how much I learn"). Students Social Support (SSS) is an index calculated through five items related to how classmates care and support them (e.g., "In math class students want me to do my best in math work"). Based on the conceptualization of attitudes as positive or negative affect associated with a certain subject [9], in this research Student Attitudes towards math is a five item index relating to how students feel in math class and when performing math school tasks (e.g., "When the teacher questions me about math I feel good"). All items were scored on a 6-point scale ranging from 1 ("Never") to 6 ("Always").

Reliability coefficients were .81 for SSS, .95 for TSS and .84 for Attitudes.

\section{Results}

The results presented focus on two main vectors. Firstly the descriptive statistics of participants' attitudes, motivation and perceived social support towards mathematics, and also their differences considering gender, grade, and math performance were analyzed. Secondly a hierarchical analysis using structural equation modeling, utilizing three blocks of variables, was carried out. These blocks were background, motivation, and support-related variables.

Descriptive statistics of students' perceived social support, motivations, and attitudes toward mathematics are presented in Table 2 in relation to gender, grades, and math performance. Attitude scores vary between 3.37 and 4.11 when different groups organized according to gender, cycle and math performance are considered. These results are above the middle point of the scale, which show that, overall, those students present positive attitudes towards mathematics.

When considering motivation dimensions we can see that scores are near the midpoint of the scale, and some differences are introduced when considering gender, study cycle, and achievement. These scores show that younger students (2nd Cycle) present higher motivational scores when compared with their older colleagues. The same pattern is presented with achievement, since students with low marks present lower scores in all dimensions when compared to medium and good achievers, and good achievers have the highest scores.

With regard to social support, the results are almost similar across gender. In looking at grade and achievement one can see that younger students perceived greater support from their math teachers. Better achievers also seem to feel 
TABLE 2: Descriptive statistics for attitudes towards mathematics by gender, study cycle and math achievement.

\begin{tabular}{|c|c|c|c|c|c|c|c|c|c|c|c|c|c|c|c|c|c|}
\hline & & \multicolumn{4}{|c|}{ Gender } & \multicolumn{6}{|c|}{ Study cycle } & \multicolumn{6}{|c|}{ Math achievement } \\
\hline & & \multicolumn{2}{|c|}{ Boys } & \multicolumn{2}{|c|}{ Girls } & \multicolumn{2}{|c|}{ 2nd Cycle } & \multicolumn{2}{|c|}{ 3rd Cycle } & \multicolumn{2}{|c|}{ Secondary } & \multicolumn{2}{|c|}{ Low } & \multicolumn{2}{|c|}{ Fair } & \multicolumn{2}{|c|}{ Good } \\
\hline & & $M$ & $\mathrm{SD}$ & $M$ & $\mathrm{SD}$ & $M$ & SD & $M$ & $\mathrm{SD}$ & $M$ & $\mathrm{SD}$ & $M$ & $\mathrm{SD}$ & $M$ & $\mathrm{SD}$ & $M$ & $\mathrm{SD}$ \\
\hline \multirow{4}{*}{ Motivation } & Attitudes & 3.72 & 1.25 & 3.70 & 1.26 & 4.11 & 1.30 & 3.67 & 1.28 & 3.37 & 1.04 & 3.38 & 1.30 & 3.62 & 1.17 & 3.99 & 1.23 \\
\hline & Value/utility & 4.16 & 1.48 & 4.46 & 1.35 & 4.96 & 1.13 & 3.84 & 1.54 & 4.43 & 1.09 & 4.0 & 1.32 & 4.32 & 1.34 & 4.43 & 1.51 \\
\hline & Perceived choice & 3.67 & 1.31 & 3.90 & 1.33 & 4.0 & 1.44 & 3.69 & 1.36 & 3.72 & 1.07 & 3.52 & 1.27 & 3.65 & 1.28 & 4.04 & 1.34 \\
\hline & Perceived competence & 3.65 & 1.27 & 3.42 & 1.16 & 3.82 & 1.17 & 3.5 & 1.3 & 3.3 & 0.86 & 3.07 & 1.18 & 3.47 & 0.91 & 3.88 & 1.25 \\
\hline \multirow{2}{*}{ Social support } & Teacher social support & 4.33 & 1.52 & 4.60 & 1.43 & 5.03 & 1.12 & 4.06 & 1.65 & 4.69 & 1.19 & 4.31 & 1.52 & 4.53 & 1.37 & 4.52 & 1.54 \\
\hline & Students social support & 3.45 & 1.14 & 3.62 & 1.09 & 3.32 & 1.1 & 3.67 & 1.12 & 3.49 & 1.10 & 3.51 & 1.15 & 3.52 & 1.14 & 3.56 & 1.1 \\
\hline
\end{tabular}

that they have more support from their teachers. Students social support scores are very similar when comparing students according to gender, cycle, or achievement.

In order to clarify differences in means, with regard to attitudes toward mathematics (Table 2) we performed a univariate analyses of variance (ANOVA) with gender, grades, and math achievement as fixed factors. No gender effect was identified, although Cycle $(F(2,1701)=41.904, P<.001)$, and math achievement $(F(2,1701)=61.075, P<.001)$ introduce significant effects in attitudes towards mathematics. Interaction effects were also identified for Gender* Cycle $(F(2,1701)=5.999, P=.003)$ and Cycle*achievement $(F(2,1701)=14.441, P<.001)$. Multiple comparisons with a Tukey post hoc test show that attitudes towards mathematics became less positive as schooling continues $(P<.001$, from 2nd to 3rd Cycles; $P<.001$, 3rd Cycle to Secondary school). A similar lowering tendency with regard to Math achievement was confirmed, with lower achieving students having less positive attitudes than Medium and Good students, and also with Medium students differing from Good students in their attitudes (low/medium $P<$ .005 ; low/good $P<.001$; medium/good $P<.001$ ).

An interaction effect between gender and Cycle on attitudes towards mathematics (Figure 1) is evidenced by the different pattern presented by girls through schooling, relative to boys. Whereas girls present a systematic decline in attitudes toward mathematics with their progression through schooling (post hoc comparisons with Tuckey test $P<.001$ ), boys present less positive attitudes towards mathematics in the 3rd Cycle and Secondary school level than in the 2nd Cycle $(P<.001)$ but boys in the 3rd Cycle and Secondary present similar attitudes towards mathematics.

The interaction effect between Cycle and achievement on attitudes towards mathematics is presented in Figure 2. Low achievers show an inverted $U$ shape curve, becoming less positive in their attitudes towards mathematics from the 3rd Cycle to the Secondary school level $(P=.002)$, whereas medium achievers adopt more negative attitudes towards mathematics the further they advance in their schooling $(P<.001$ for the differences between Secondary school level and 2nd and 3rd Cycles; $P=.08$ for the difference between 2nd and 3rd Cycles). Good achievers show a greater decrease, between the 2nd and 3rd Cycles $(P<.001)$, in attitudes towards mathematics and then a small rise when they are at Secondary school (albeit not significant). From

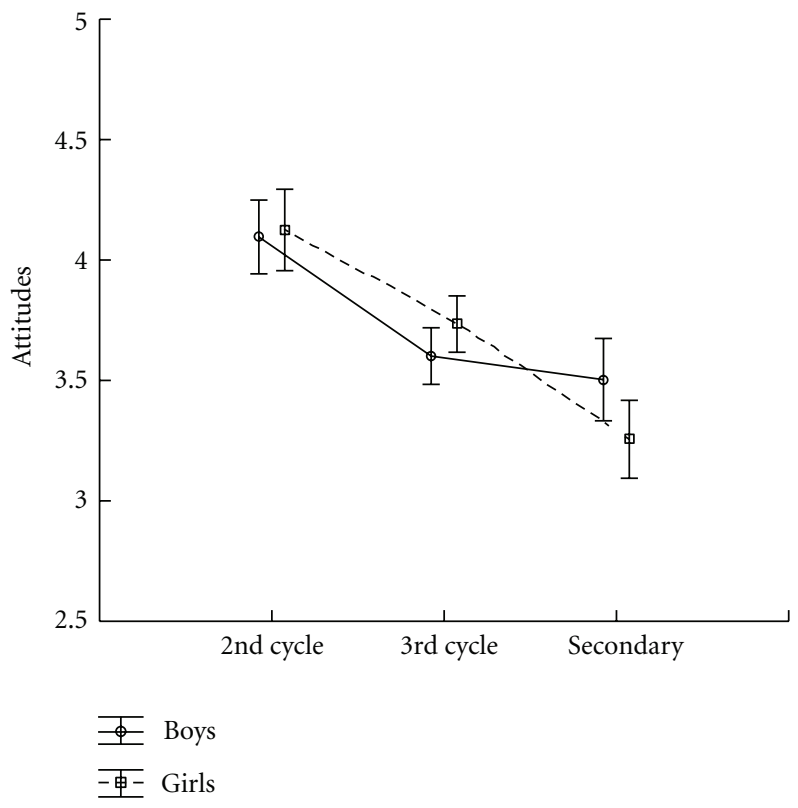

FIGURE 1: Interaction effect between gender and cycle on attitudes towards mathematics.

the point of view of study cycle, this interaction effect is evidenced by the fact that 3rd Cycle students present similar levels of attitudes towards mathematics, independent of their achievement status, which does not happen in the other cycles of Studies $(P<.001$ for the differences between the three levels of achievement at the 2nd Cycle, and between good achievers and the other two groups at Secondary school level).

Bivariate correlations were computed to examine relations among background, motivational, attitudes and social support variables and are presented in Table 3.

The correlation scores are in general positive, significant, but not very strong. The most positive highlights the associations between attitudes and motivation or support variables. Negative correlations were found when considering Cycle pointing to the idea that older students present less positive attitudes lower levels of motivation and feel less support from their teachers.

In order to test the relationships between background, motivational and social support-related variables with attitudes towards mathematics, we carried out a hierarchical 
TABLE 3: Correlations between study variables.

\begin{tabular}{|c|c|c|c|c|c|c|c|c|}
\hline & Gender & $\begin{array}{c}\text { Math } \\
\text { achievement }\end{array}$ & Cycle & $\begin{array}{l}\text { Value/ } \\
\text { Utility }\end{array}$ & $\begin{array}{l}\text { Perceived } \\
\text { Choice }\end{array}$ & $\begin{array}{c}\text { Perceived } \\
\text { Competence }\end{array}$ & $\begin{array}{l}\text { Teacher social } \\
\text { support }\end{array}$ & $\begin{array}{l}\text { Students social } \\
\text { support }\end{array}$ \\
\hline Gender & - & & & & & & & \\
\hline Math achievement & .028 & - & & & & & & \\
\hline Cycle & $.055^{*}$ & .013 & - & & & & & \\
\hline Value/utility & $.086^{* *}$ & $.116^{* *}$ & $-.140^{* *}$ & - & & & & \\
\hline Perceived choice & $.087^{* *}$ & $.178^{* *}$ & $-.079^{* *}$ & $.453^{* *}$ & - & & & \\
\hline Perceived competence & $-.096^{* *}$ & $.289^{* *}$ & $-.157^{* *}$ & $.431^{* *}$ & $.326^{* *}$ & - & & \\
\hline Teacher social support & $.090^{* *}$ & .040 & $-.087^{* *}$ & $.706^{* *}$ & $.319^{* *}$ & $.257^{* *}$ & - & \\
\hline Students social support & $.076^{* *}$ & .020 & $.055^{*}$ & $.228^{* *}$ & $.127^{* *}$ & $.121^{* *}$ & $.287^{* *}$ & - \\
\hline Attitudes & -.007 & $.212^{* *}$ & $-.209^{* *}$ & $.549^{* *}$ & $.562 * *$ & $.529^{* *}$ & $.494^{* *}$ & $.299 * *$ \\
\hline
\end{tabular}

* Correlation is significant at the 0.05 level; ${ }^{* *}$ correlation is significant at the 0.01 level.

Gender codification: (1) boys; (2) girls.

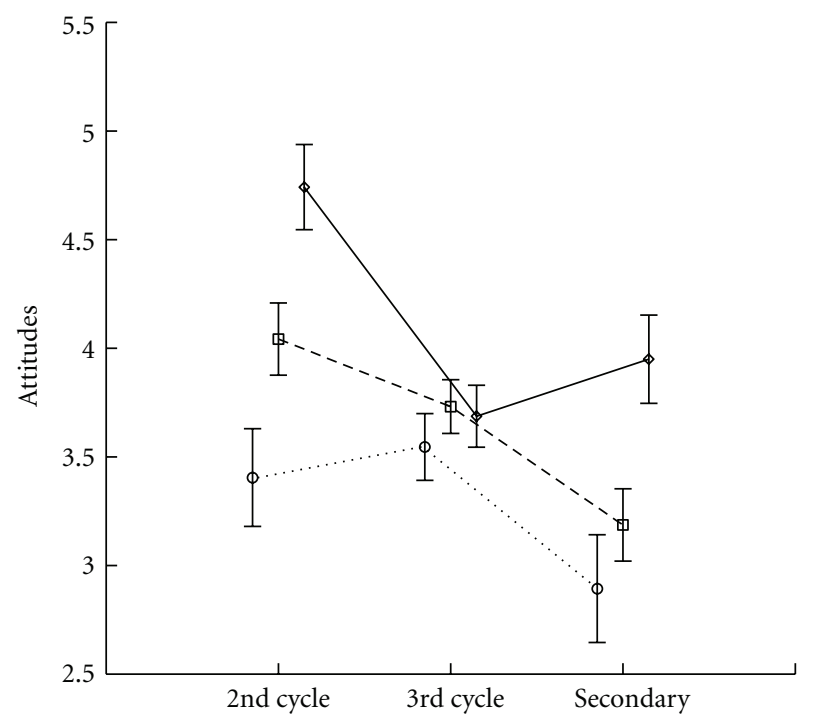

Math achievement

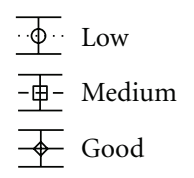

Figure 2: Interaction effect between math achievement and cycle on attitudes towards mathematics.

analysis using structural equation modeling with Amos 20.0. In this analysis, we have taken three blocks of variables into consideration (Table 4). The first block, named background variables, includes gender, grade, and performance in mathematics; the second block includes mathematics motivation dimensions and the third block looks at social support variables. Because all the variables in the second and third block are self-reported, potentially introducing bias with spurious relations, the recommendations of Podsakoff et al. [37] were followed in the treatment. Thus, in order to control for method bias due to the fact that all measures are self-reported, in models 2 and 3 we added a common latent factor with all the items of all measures loading in this factor. In the three models tested we only considered the relationships between each variable and attitudes, discarding other possible relationships among them. All the models tested presented acceptable fit (Model 1: AGFI -.98, CFI -.96 , TLI -.94, RMSEA -.06; Model 2: GFI -.92, CFI -.92, TLI -.89, RMSEA -.076; Model 3: GFI -.91, CFI - .93, TLI -.92 , RMSEA -.06).

In all models the three blocks of variables (background, motivation, and social support) are significant contributors to Attitudes. Background variables explained $8.9 \%$ of the variance and Math Motivation variables explained a further $31.3 \%$. When adding Support variables (teacher and students) the model gains a further $24.1 \%$ in the variance explained. Therefore the total amount of variation in Attitudes explained by all the variables included in the final model is $64.3 \%$. We can also verify in Model 3 that among Background variables only Cycle has a significant effect on Attitudes $(\beta=-.055 P<.001)$. Among motivational variables Perceived Choice and Perceived Competence relate significantly to attitudes $(\beta=.451$ and $\beta=.495$ resp. $)$, whereas both dimensions of social support are positively related to attitudes towards Maths. The contribution of both is also significant for this model $(P<.001)$, with a higher contribution of the perception of teacher social support ( $\beta=.307)$, when compared with the perception of support provided by peers $(\beta=.246)$.

\section{Discussion}

This paper first sought to characterize attitudes towards mathematics in students from 5th to 12 th grade and to analyze the effects of gender, cycle, and math performance on these attitudes. Results showed that, in general, the students had positive attitudes towards mathematics, although scores were not very high and distributed mostly around the midpoint. Despite this overall positive attitude towards mathematics the scenario changes when we consider Cycle. Cycle effects are significant and mean that during schooling, attitudes towards mathematics become less positive. In fact, students in the 2nd Cycle present attitudes towards 
TABLe 4: Parameter estimates and associated standard errors hierarchical analysis on attitudes towards mathematics.

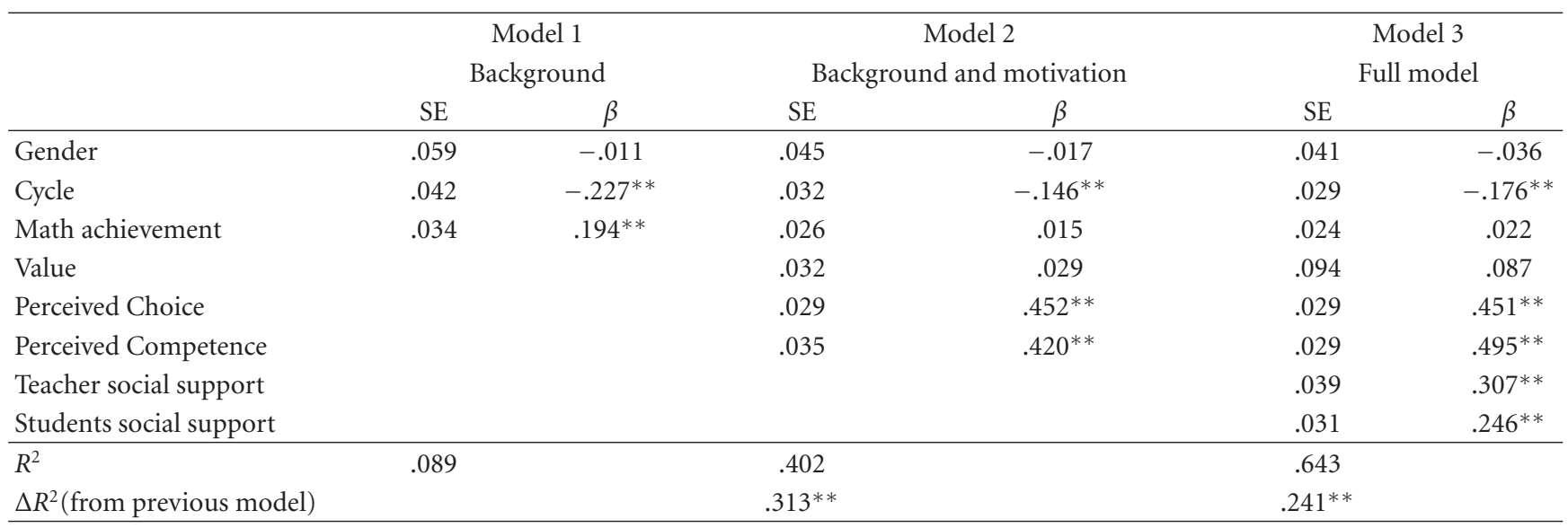

${ }^{*} P<0.01,{ }^{* *} P<0.001$.

mathematics which are clearly positive, whereas students in Secondary school exhibit values below the midpoint of the scale. Considering that attitudes towards learning can be related to motivation [38] the decrease in attitudes towards mathematics can be associated with the overall decrease in intrinsic motivation, competence-related beliefs, interest and task values that occur during adolescence [39-41]. This decline is experienced in math in particular [41-43]. Motivation theorists have argued that during adolescence interests are directed towards other fields of experience which could explain the fall in school-related attitudes and interest. An additional explanation is related to the organization of the math curriculum which becomes more demanding as students move through grade levels, requiring increasingly abstract levels of understanding [44]. However, challenge is also an important feature of motivation [45] inasmuch as the challenge is not that great that it would be experienced as overwhelming, leading to feelings of helplessness. Interaction effects between Cycle and Math Achievement seem to provide some support to this explanation. Medium achievers show a gradual decrease in attitudes towards mathematics across the school years whereas low achievers present a slight increase from 2nd to 3rd Cycles and a decline in the transition from 3rd Cycle to Secondary school. An inverse pattern is presented by good achievers. The differences in the pattern of good and low achievers seems to support the hypothesis of differences in the way the challenges set by math learning are experienced by students belonging to different achievement groups. For good achievers mathematical tasks are likely faced as real challenges which could increase intrinsic motivation, raising the sense of competence when the tasks are solved, and leading to the development of positive attitudes towards math. Conversely, for low achievers math tasks are likely experienced as unsurpassable obstacles that will be won infrequently, producing low self-belief in competence and negative attitudes towards mathematics.

Gender-related attitudes towards mathematics seem to be identical. This finding corroborates the results of other research that claims that boys and girls present very similar attitudes towards mathematics $[3,5,6,15,16]$. However, results also show an interaction effect between gender and study cycle that results in a systematic decline in attitudes towards mathematics along schooling. This counteracts the pattern presented by boys which showed a decrease in their attitudes from the 2nd to the 3rd Cycles but which then stabilized. This progressive decline in attitudes by girls can be explained with reference to gender stereotypes [46]. Traditionally math is viewed as a male-dominated domain which is evident in career choices and jobs [47]. Studies in stereotyping and development in adolescence support the idea of gender intensification during middle and late adolescence accompanied by less flexibility to stereotyping [46]. This leads to the assumption of roles according to gender, assuming gender-type interests which could explain the less positive attitudes towards mathematics exhibited by girls at Secondary school.

Our findings concerning the relationship between math achievement and attitudes towards mathematics are consistent with research showing that good achievers develop more positive attitudes than lower achievers $[2,4,14,16,20]$. Achievement is usually related to self-belief in competence $[41,48]$ and self-belief in competence can be related to attitudes towards math [38], which suggests that when students succeed at a math task, it increases their sense of competence and this may promote more positive attitudes.

The hierarchical analysis using structural equation modeling extends the findings of this study. The first model tested accounts for less than $9 \%$ of variance in attitudes, showing that the effects of Gender, Cycle and Math Achievement are relatively small. Moreover, the contribution of Cycle and achievement for attitudes towards mathematics diminished when psychological variables of students were added. In fact, motivational variables increase the amount of variance explained in attitudes, showing a close relationship between key features of intrinsic motivation and attitudes towards mathematics. Authors involved in intrinsic motivation research have shown that students learn more effectively when they are interested and when they enjoy what they are learning $[6,32]$. According to these authors students who feel competent and self-determinate in an ongoing, continuous 
way, increase their intrinsic motivation. Likewise, positive attitudes towards mathematics may also increase since they have been conceived as positive or negative emotional dispositions toward a subject and positive emotion is, in general, perceived as pleasurable [9]. Our findings support these ideas revealing that the positive predictors of intrinsic motivation (Perceived Competence and Perceived Choice) and are more strongly related to attitudes towards mathematics. Adding learning environment-related variables to the model also produces an increase in the explanation of the variability of attitudes.

Among the variables considered (teacher support and peer support), teacher support shows closer relationships to attitudes. In the final model, this is the third strongest relationship which shows the importance of teachers in the development of positive attitudes towards math.

Despite the importance of teacher support as a significant predictor of attitudes, we cannot neglect the effect of peer support identified in our data and in research by Fraser and Kahle [8]. However, the improvement in attitudes is likely to be more significant when taking into consideration different environments, but the main contribution is determined in the class environment. Research on this topic has shown that teacher support with regard to autonomy affected student motivation, among other aspects, $[49,50]$ and that different pedagogical goals also explained variations in student math motivation [51]. Taken together, these findings highlight the role of the teacher in supporting student learning, attitudes and even motivation and have some implications for education and instructional practices. As Aunola et al. [51] have shown, teacher goals may influence child motivation and attitudes not only through their instructional practices and the tasks they propose to students, but also through the messages they send out about learning in general. In this sense if teachers create situations that promote pleasure, are seen as self-determinate and students feel competent; intrinsic motivation can increase [32, 33], and this may also promote positive attitudes towards mathematics. A teacher who is supportive to students [52], who shapes student expectations about learning in a positive way [53], who sets meaningful tasks which are somewhat, but not excessively challenging $[54,55]$, and promotes cooperative learning environments $[23,56]$ will probably stimulate intrinsic motivation in their students and, as a corollary, may contribute to the development of more positive attitudes towards math.

The results presented here suggest strong relationships between motivation and support related variables with attitudes. However, the cross-sectional natures of the design used do not allow us to infer causal relationships between the variables. Therefore, for the interpretation of the results we cannot say that motivational and social context factors influence student's attitudes, although we cannot rule out that variables closely related to motivation and social support in the classroom have a significant relationship to attitudes. The use of multiple assessments over time could provide a clearer view of the causal relations between variables. Furthermore, a longitudinal design could help us to achieve a better understanding not only of the changes in time, but also of the development of attitudes toward mathematics and the effects of other variables such as background, motivation, and social support. Likewise, the use of a small group study and the monitoring of the group including multiple assessments over time, also using qualitative data, could provide a deeper comprehension of the heterogeneity of attitudes among subjects and allows for the researcher to take into account the effects of aging on the group. As suggested by Hannula [57] qualitative data collected from observations, interviews, and case studies can be useful in studying attitudes more accurately and to recognize possible factors behind the characteristics of attitudes and any changes that take place.

Another limitation of this study is the fact that only selfreport measures were used. It is possible to reduce the bias introduced by single-source data using appropriate statistical procedures [37] as we have done in this research. Although, a worthwhile addition to a future study to overcome this problem would be to incorporate more than one data source, namely, data collected through classroom observations and teacher-reports.

A good deal of research has been conducted on attitudes towards mathematics, but most of the analyses used have focused on how specific variables influence or are related to attitudes, considering these variables in an isolated way. In order to have a more complex perspective towards this topic, the present research has attempted to examine the combined effects of the individual, social contextual, and motivational variables on attitudes toward mathematics. The results of the integration of data provide a more in-depth understanding of the different variables which allow for the exploration of different routes in promoting positive attitudes toward mathematics. On the other hand, researches concerning the relationship between motivation, social support from teachers and peers, and attitudes are scarce. In this regard, our data emphasizes the importance of these variables when trying to understand attitudes toward mathematics.

Lack of student motivation and engagement in academic work is an issue of concern amongst teachers. Since our findings confirm that attitudes are deeply related to motivation and social support, we believe that developing strategies in educational contexts, to improve teacher support and student engagement could be of vital importance in improving not only attitudes but also mathematical performance among students throughout their schooling.

\section{Acknowledgment}

This research was supported by Grants from the Science and Technology Foundation (POCI 2010).

\section{References}

[1] A. Serrão, C. Ferreira, and H. Diniz de Sousa, PISA 2009: Competências dos alunos portugueses: Sintese de resultados, GAVE, Ministry of Education, Lisbon, Portugal, 2010.

[2] K. Singh, M. Granville, and S. Dika, "Mathematics and science achievement: effects of motivation, interest, and academic engagement," Journal of Educational Research, vol. 95, no. 6, pp. 323-332, 2002. 
[3] D. Köğce, C. Yıldız, M. Aydın, and R. Altındağ, "Examining elementary school students' attitudes towards mathematics in terms of some variables," Procedia, vol. 1, no. 1, pp. 291-295, 2009.

[4] M. Mato and E. De la Torre, "Evaluación de las actitudes hacia las matemáticas y el rendimiento académico," PNA, vol. 5, no. 1, pp. 197-208, 2010.

[5] L. Mohamed and H. Waheed, "Secondary students' attitude towards mathematics in a selected school of Maldives," International Journal of Humanities and Social Science, vol. 1, no. 15 , pp. 277-281, 2011.

[6] M. Nicolaidou and G. Philippou, "Attitudes towards mathematics, self-efficacy and achievement in problem solving," in European Research in Mathematics Education III, M. A. Mariotti, Ed., pp. 1-11, University of Pisa, Pisa, Italy, 2003.

[7] B. Eshun, "Sex-differences in attitude of students towards Mathematics in secondary schools," Mathematics Connection, vol. 4, pp. 1-13, 2004.

[8] B. J. Fraser and J. B. Kahle, "Classroom, home and peer environment influences on student outcomes in science and mathematics: an analysis of systemic reform data," International Journal of Science Education, vol. 29, no. 15, pp. 18911909, 2007.

[9] R. Zan and P. Martino, "Attitude toward mathematics: overcoming the positive/negative dichotomy," in Beliefs and Mathematics, B. Sriraman, Ed., The Montana Mathematics Enthusiast: Monograph Series in Mathematics Education, pp. 197-214, Age Publishing \& The Montana Council of Teachers of Mathematics, Charlotte, NC, USA, 2008.

[10] T. Scafidi and K. Bui, "Gender similarities in math performance from middle school through high school," Journal of Instructional Psychology, vol. 37, no. 3, pp. 252-255, 2010.

[11] S. M. Lindberg, J. S. Hyde, J. L. Petersen, and M. C. Linn, "New trends in gender and mathematics performance: a metaanalysis," Psychological Bulletin, vol. 136, no. 6, pp. 1123-1135, 2010.

[12] S. Skaalvik and E. M. Skaalvik, "Gender differences in math and verbal self-concept, performance expectations, and motivation," Sex Roles, vol. 50, no. 3-4, pp. 241-251, 2004.

[13] K. Asante, "Secondary students' attitudes towards mathematics," IFE PsychologIA, vol. 20, no. 1, pp. 121-133, 2012.

[14] K. Sanchez, L. Zimmerman, and R. Ye, "Secondary students' attitudes toward mathematics," Academic Exchange Quarterly, vol. 8, no. 2, pp. 56-60, 2004.

[15] X. Ma and N. Kishor, "Assessing the relationship between attitude toward mathematics and achievement in mathematics: a meta-analysis," Journal for Research in Mathematics Education, vol. 28, no. 1, pp. 26-47, 1997.

[16] S. Georgiou, P. Stavrinides, and T. Kalavana, "Is Victor better than Victoria at maths?" Educational Psychology in Practice, vol. 23, no. 4, pp. 329-342, 2007.

[17] Y. Etsey and S. Snetzler, "A Meta-analysis of gender differences in student attitudes toward mathematics," in Proceedings of the Annual Meeting of the American Educational Research Association, 1998.

[18] J. Hyde, E. Fennema, M. Ryan, L. Frost, and C. Hopp, "Gender comparisons of mathematics attitudes and affect-A metaanalysis," Psychology of Woman Quarterly, vol. 14, no. 3, pp. 299-324, 1990.

[19] H. Schofield, "Sex, grade, level, and relationships between mathematics attitude and achievement in children," Journal of Educational Research, vol. 75, no. 5, pp. 280-284, 1982.

[20] A. A. Lipnevich, C. MacCann, S. Krumm, J. Burrus, and R. D. Roberts, "Mathematics attitudes and mathematics outcomes of US and Belarusian middle school students," Journal of Educational Psychology, vol. 103, no. 1, pp. 105-118, 2011.

[21] T. Akey, "School context, students attitudes and behavior and academic achievement: an exploratory analysis," Tech. Rep., MDRC, 2006.

[22] S. Maat and E. Zakaria, "The learning environment, teacher's factor and students attitudes towards mathematics amongst engineering technology students," International Journal of Academic Research, vol. 2, no. 2, pp. 16-20, 2010.

[23] W. Vaughan, "Effects of cooperative learning on achievement and attitude among students of color," Journal of Educational Research, vol. 95, no. 6, pp. 359-364, 2002.

[24] D. Rawnsley and D. Fisher, "Learning environments in mathematics classrooms and their associations with students' attitudes and learning," in Proceedings of the Australian Association for Research in Education Conference, Adelaide, Australia, 1998.

[25] R. Pekrun, T. Goetz, W. Titz, and R. P. Perry, "Academic emotions in students' self-regulated learning and achievement: a program of qualitative and quantitative research," Educational Psychologist, vol. 37, no. 2, pp. 91-105, 2002.

[26] T. Goetz, R. Pekrun, N. Hall, and L. Haag, "Academic emotions from a social-cognitive perspective: antecedents and domain specificity of students' affect in the context of Latin instruction," British Journal of Educational Psychology, vol. 76, no. 2, pp. 289-308, 2006.

[27] A. Wigfield, "Reading motivation: a domain-specific approach to motivation," Educational Psychologist, vol. 32, no. 2, pp. 59$68,1997$.

[28] J. Guthrie and K. Knowles, "Promoting reading motivation," in Literacy and Motivation. Reading Engagement in Individuals and Groups, L. Verhoven and C. Snow, Eds., Lawrence Erlbaum Associates, Hillsdale, NJ, USA, 2001.

[29] B. Hemmings and R. Kay, "Prior achievement, effort, and mathematics attitude as predictors of current achievement," Australian Educational Researcher, vol. 37, no. 2, pp. 41-58, 2010.

[30] A. Reynolds and H. Walberg, "A structural model of high school mathematics outcomes," Journal of Educational Research, vol. 85, no. 3, pp. 150-158, 1992.

[31] L. Mata, V. Monteiro, and F. Peixoto, "Intrinsic Motivation Inventory: psychometric properties in its application in the context of learning Portuguese and Mathematics," In press.

[32] E. L. Deci and R. M. Ryan, "The "what" and "why" of goal pursuits: human needs and the self-determination of behavior," Psychological Inquiry, vol. 11, no. 4, pp. 227-268, 2000.

[33] E. Deci and R. Ryan, Intrinsic Motivation and Self-Determination in Human Behavior, Plenum, New York, NY, USA, 1985.

[34] SDT (n.d), http://www.psych.rochester.edu/SDT/measures/ IMI_description.php.

[35] J. C. Nunnaly, Psychometric Theory, McGraw-Hill, New York, NY, USA, 1978.

[36] L. Mata, V. Monteiro, and F. Peixoto, "Escala de clima de sala de aula," in Avaliação psicológica: Instrumentos validados para a população portuguesa, C. Machado, M. M. Gonçalves, M. R. Simões, and L. S. Almeida, Eds., vol. 4, Almedina, Coimbra, Portugal.

[37] P. M. Podsakoff, S. B. MacKenzie, J. Y. Lee, and N. P. Podsakoff, "Common method biases in behavioral research: a critical review of the literature and recommended remedies," Journal of Applied Psychology, vol. 88, no. 5, pp. 879-903, 2003.

[38] J. Green, G. Liem, A. Martin, S. Colmar, H. Marsh, and D. McInerney, "Academic motivation, self-concept, engagement, 
and performance in high school: key processes from a longitudinal perspective," Journal of Adolescence, vol. 35, no. 5, pp. 1111-1122, 2012.

[39] M. Covington and E. Dray, "The developmental course of achievement motivation: a need-based approach," in Development of Achievement Motivation, A. Wigfield and J. S. Eccles, Eds., pp. 33-56, Academic Press, San Diego, Calif, USA, 2002.

[40] J. E. Jacobs, S. Lanza, D. W. Osgood, J. S. Eccles, and A. Wigfield, "Changes in children's self-competence and values: gender and domain differences across grades one through twelve," Child Development, vol. 73, no. 2, pp. 509-527, 2002.

[41] A. Wigfield, J. Eccles, U. Schiefele, R. Roeser, and P. DavisKean, "Development of achievement motivation," in Handbook of Child Psychology, N. Eisenberg, W. Damon, R. M. Lerner, N. Eisenberg, W. Damon, and R. M. Lerner, Eds., vol. 3 of Social, Emotional, and Personality Development, pp. 933 1002, John Wiley \& Sons, Hoboken, NJ, USA, 6th edition, 2006.

[42] J. A. Fredricks and J. S. Eccles, "Children's competence and value beliefs from childhood through adolescence: growth trajectories in two male-sex-typed domains.," Developmental Psychology, vol. 38, no. 4, pp. 519-533, 2002.

[43] A. E. Gottfried, G. A. Marcoulides, A. W. Gottfried, and P. H. Oliver, "A latent curve model of parental motivational practices and developmental decline in math and science academic intrinsic motivation," Journal of Educational Psychology, vol. 101, no. 3, pp. 729-739, 2009.

[44] J. Turner and D. Meyer, "Understanding motivation in mathematics: what is happening in classrooms?" in Handbook of Motivation at School, K. R. Wenzel, A. Wigfield, K. R. Wenzel, and A. Wigfield, Eds., pp. 527-552, Routledge/Taylor \& Francis Group, New York, NY, USA, 2009.

[45] S. Miller, "How high- and low-challenge tasks affect motivation and learning: implications for struggling learners," Reading and Writing Quarterly: Overcoming Learning Difficulties, vol. 19, no. 1, pp. 39-57, 2003.

[46] D. N. Ruble, C. Martin, and S. Berenbaum, "Gender development," in Handbook of Child Psychology, N. Eisenberg, W. Damon, R. M. Lerner, N. Eisenberg, W. Damon, and R. M. Lerner, Eds., vol. 3 of Social, Emotional, and Personality Development, pp. 858-932, John Wiley \& Sons, Hoboken, NJ, USA, 6th edition, 2006.

[47] C. Leaper, T. Farkas, and C. S. Brown, "Adolescent girls' experiences and gender-related beliefs in relation to their motivation in math/science and English," Journal of Youth and Adolescence, vol. 41, no. 3, pp. 268-282, 2012.

[48] F. Peixoto and L. S. Almeida, "Self-concept, self-esteem and academic achievement: strategies for maintaining self-esteem in students experiencing academic failure," European Journal of Psychology of Education, vol. 25, no. 2, pp. 157-175, 2010.

[49] V. F. Filak and K. M. Sheldon, "Teacher support, student motivation, student need satisfaction, and college teacher course evaluations: testing a sequential path model," Educational Psychology, vol. 28, no. 6, pp. 711-724, 2008.

[50] N. Gillet, R. J. Vallerand, and M. A. K. Lafrenière, "Intrinsic and extrinsic school motivation as a function of age: the mediating role of autonomy support," Social Psychology of Education, vol. 15, no. 1, pp. 77-95, 2012.

[51] K. Aunola, E. Leskinen, and J. E. Nurmi, "Developmental dynamics between mathematical performance, task motivation, and teachers' goals during the transition to primary school," British Journal of Educational Psychology, vol. 76, no. 1, pp. 21-40, 2006.
[52] C. P. Niemiec and R. M. Ryan, "Autonomy, competence, and relatedness in the classroom: applying self-determination theory to educational practice," Theory and Research in Education, vol. 7, no. 2, pp. 133-144, 2009.

[53] J. Brophy, Motivating Students to Learn, Rowtledge, New York, NY, USA, 2010.

[54] M. K. Alderman, Motivation for Achievement. Possibilities for Teaching and Learning, Lawrence Erlbaum Associates Publishers, Mahwah, NJ, USA, 2004.

[55] D. Stipek, Motivation to Learn. Integrating Theory and Practice, Allyn and Bacon, Boston, Mass, USA, 2002.

[56] G. Ghaith, "The relationship between forms of instruction, achievement and perceptions of classroom climate," Educational Research, vol. 45, no. 1, pp. 83-93, 2003.

[57] M. S. Hannula, "Attitude towards mathematics: emotions, expectations and values," Educational Studies in Mathematics, vol. 49 , no. 1 , pp. $25-46,2002$. 


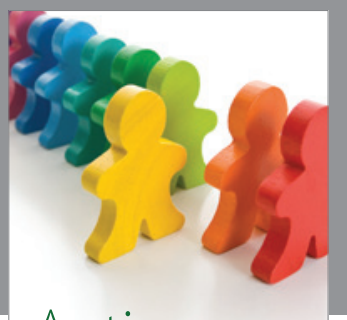

Autism

Research and Treatment
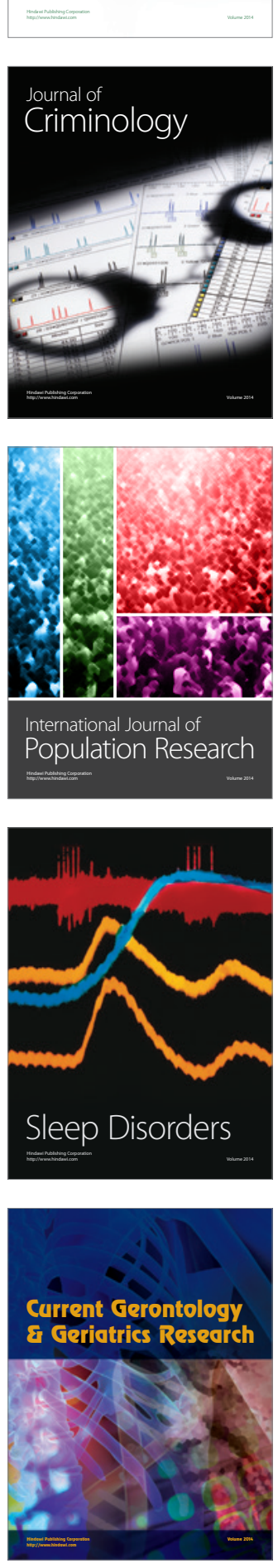
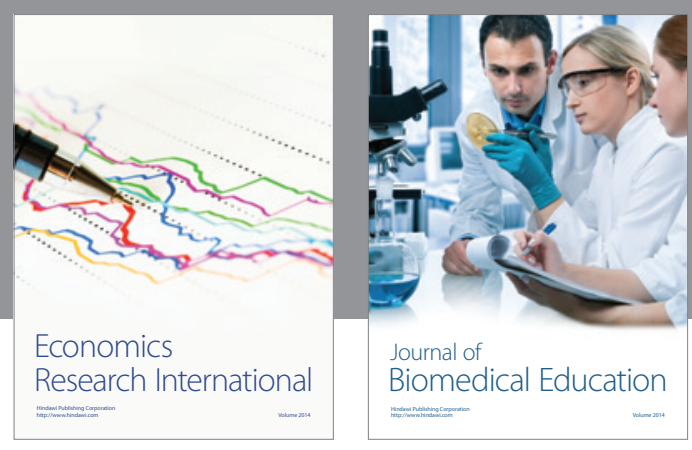

Journal of

Biomedical Education

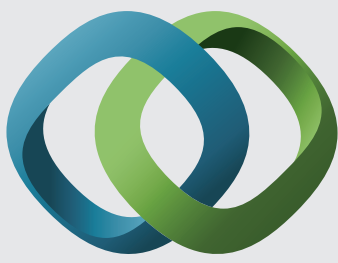

\section{Hindawi}

Submit your manuscripts at

http://www.hindawi.com
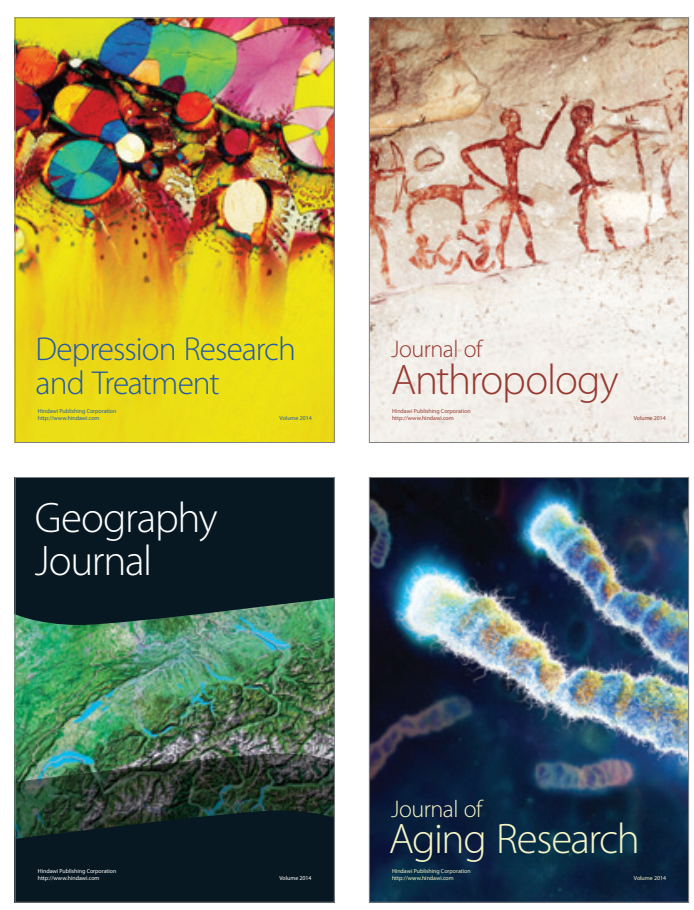

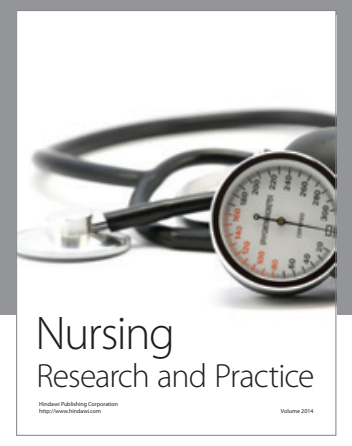

Nursing

Research and Practice

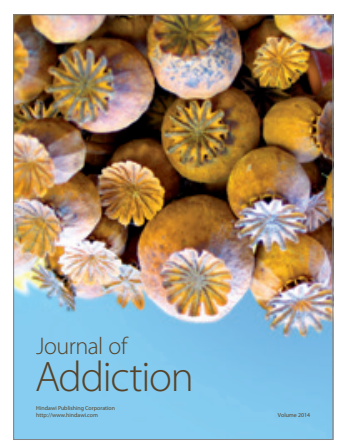

Child Development

Research

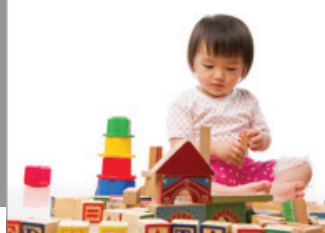

迥
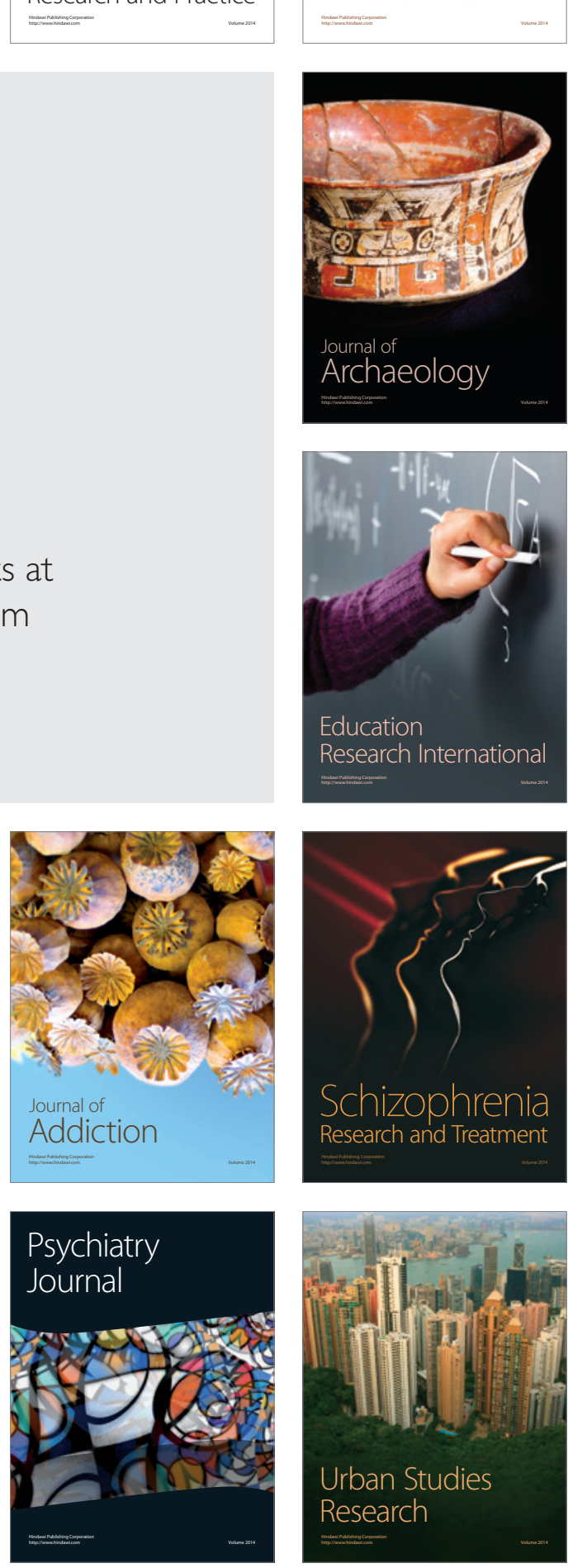